\title{
Changes in Menopausal Risk Factors in Early Postmenopausal Osteopenic Women After 13 Months of High-Intensity Exercise: The Randomized Controlled ACTLIFE-RCT
}

This article was published in the following Dove Press journal:

Clinical Interventions in Aging

\author{
Michael Hettchen (D) \\ Simon von Stengel ${ }^{\prime}$ \\ Matthias Kohl (iD ${ }^{2}$ \\ Marie H Murphy $\mathbb{D D}^{3}$ \\ Mahdieh Shojaa' \\ Mansour Ghasemikaram' \\ Laura Bragonzoni ${ }^{4}$ \\ Francesco Benvenuti ${ }^{4}$ \\ Claudio Ripamonti (iD ${ }^{5}$ \\ Maria Grazia Benedetti ${ }^{6}$ \\ Mikko Julin ${ }^{7}$ \\ Tapani Risto ${ }^{7}$ \\ Wolfgang Kemmler (D) \\ 'Institute of Medical Physics, Friedrich- \\ Alexander University Erlangen-Nürnberg, \\ Erlangen, Germany; ${ }^{2}$ Department of \\ Medical and Life Sciences, University of \\ Furtwangen, Schwenningen, Germany; \\ ${ }^{3}$ Doctoral College, Ulster University, \\ Newtownabbey, Antrim, Northern \\ Ireland, UK; ${ }^{4}$ University of Bologna, \\ Campus Rimini, Rimini, Italy; ${ }^{5}$ Center for \\ Osteoporosis and Bone Metabolic \\ Disease, Rizzoli Orthopaedic Institute, \\ Bologna, Italy; ' ${ }^{6}$ hysical Medicine and \\ Rehabilitation Unit, IRCCS - Rizzoli \\ Orthopaedic Institute, Bologna, Italy; \\ ${ }^{7}$ Laurea University of Applied Sciences, \\ Espoo, Finland
}

Correspondence: Michael Hettchen Institute of Medical Physics, FriedrichAlexander-University Erlangen-Nürnberg, Henkestrasse 91, Erlangen 91052,

Germany

$\mathrm{Tel}+499|3|-8525883$

Fax +49 9131-8522824

Email michael.hettchen@imp.unierlangen.de

\begin{abstract}
The menopausal transition is a critical period in women's lives. Exercise might be the most promising non-pharmaceutic intervention to address the large variety of risk factors related to the pronounced estradiol decline during peri- and early-postmenopause. The aim of this study was to determine the effect of an 18-month multipurpose exercise program on risk factors and symptoms related to the menopausal transition. Fifty-four women 1-5 years postmenopause with osteopenia or osteoporosis were randomly assigned 1) to a high impact weight-bearing/high-intensity/velocity resistance training group (EG: $n=27$ ) exercising three times a week or 2) to an attendance control group (CG: $n=27)$ that performed low-intensity exercise once a week. Both groups were supplemented with cholecalciferol and calcium. The primary study endpoint was bone mineral density (BMD) at lumbar spine (LS) and total hip, secondary outcomes were lean body mass (LBM), total and abdominal body percentage, metabolic syndrome Z-Score (MetS-Z), menopausal symptoms and muscle strength and power. Due to COVID-19, the study was stopped after 13 months. We observed significant effects for BMD-LS (EG: 0.002 \pm .018 versus CG: $-.009 \pm 0.018 \mathrm{mg} / \mathrm{cm} 2, \mathrm{p}=0.027$ ) but not for BMD total hip (EG: $-0.01 \pm .016$ versus CG: $-.009 \pm 0.020 \mathrm{mg} / \mathrm{cm} 2, \mathrm{p}=0.129$ ). LBM improved significantly in the EG and decreased in the CG $(0.39 \pm 1.08$ vs $-0.37 \pm 1.34 \mathrm{~kg}, \mathrm{p}=0.026)$. Total and abdominal body fat improved significantly in the EG and was maintained in the CG $(-1.44 \pm 1.49$ vs $-0.02 \pm 1.55 \mathrm{~kg}, \mathrm{p}=0.002$ and $-1.50 \pm 2.33$ vs $0.08 \pm 2.07 \mathrm{~kg}, \mathrm{p}=0.011)$. Significant effects in favor of the EG were also determined for menopausal symptoms $(p=0.029)$, hip/leg extension strength $(p<0.001)$ and power $(p<0.001)$. However, changes of the MetS-Z did not differ significantly $(p=0.149)$ between EG and CG. In summary, with minor exceptions, we demonstrated the effectiveness of a multipurpose exercise protocol dedicated to early-postmenopausal women on various risk factors and complaints related to the menopausal transition.
\end{abstract}

Keywords: multipurpose exercise, bone mineral density, early postmenopause

\section{Introduction}

The consequences of the menopausal transition considerably affect women's lives. In addition to the psychosocial effects, ${ }^{1}$ the pronounced perimenopausal/early postmenopausal decline of estradiol (E2) ${ }^{2,3}$ frequently initiates clinical changes including accelerated bone loss, ${ }^{4}$ changes in body composition, ${ }^{5,6}$ functional declines $^{7,8}$ and/or menopausal symptoms. ${ }^{9}$ Exercise might be the most promising non-pharmaceutic intervention ${ }^{10-13}$ to address the variety of (early) menopausal 
risk factors and complaints. ${ }^{13-16}$ However, considering that exercise protocols are in general specifically aligned to one outcome or condition, the challenge of designing comprehensive exercise programs for (early-) menopausal women is obvious. We addressed the issue of designing a multipurpose exercise protocol for early-menopausal women in two earlier exercise studies; ${ }^{17,18}$ however, in the present ACTLIFE (Physical ACTivity: the tool to improve the quality of LIFE in osteoporosis people) study we additionally (1) set out to condense the exercise program to reduce the time demand and (2) particularly focused on relatively young women with osteopenia and osteoporosis who were not eligible for pharmacologic therapy due to their young age. The time-effective highintensity endurance and resistance exercise approach specifically dedicated to women in their early fifties might be considered as the unique selling point of the ACTLIFE study. Correspondingly, the aim of ACTLIFE was to determine the effect of an 18-month multipurpose exercise program on risk factors and symptoms related to the menopausal transition, with specific regard to changes in BMD.

The primary hypothesis was that the exercise group (EG) of early postmenopausal women with osteopenia and osteoporosis would show significantly higher effects on a) BMD at the LS and b) total hip compared with an attention control group (CG).

Secondary hypotheses were that the EG would show significantly higher effects on a) soft (bone free) lean body mass, b) total and c) abdominal body fat, d) cardiometabolic risk, e) menopausal symptoms and f) physical fitness compared with an attention CG.

\section{Methods}

The present work is part of the ACTLIFE project, a European Project that focuses on the development and dissemination of best practice exercise protocols for secondary and tertiary prevention of osteoporosis. The ACTLIFE consortium is comprised of scientific partner institutions from Bulgaria, Finland, Germany, Ireland and the UK, headed by the University of Bologna, Italy. The present study aimed to validate exercise recommendations derived from national guidelines and meta-analyses ${ }^{16}$ in clinical practice. The study was planned and initiated as an 18-month randomized, controlled, semi-blinded exercise trial in a parallel group design with one exercise and one attention control group. The Institute of Medical Physics (IMP), University of Erlangen-Nürnberg (FAU), Germany as the responsible partner within the ACTLIFE consortium, implemented and conducted the study. The present RCT was approved by the Ethics Committee of the FriedrichAlexander-Universität Erlangen-Nürnberg (number 118_18b) and the Federal Bureau of Radiation Protection (BfS, number Z5 - 22,462/2 - 2018-055). ACTLIFE-RCT fully complies with the Helsinki Declaration. ${ }^{19}$ All the study participants gave their written informed consent after detailed information. The RCT was registered under ClinicalTrials.gov: NCT03959995. Although we report study results after 13 months of exercise (February 2019March 2020), due to the COVID-19 induced lockdown in Bavaria, Germany on 20 March 2020, the data have to be considered as final study results.

\section{Participants}

The recruitment process of the ACTLIFE-RCT was reported previously. Briefly, using a pool of about 9600 contact data records provided by the municipality, 2500 women aged 48-60 years living independently in the area of Erlangen-Nürnberg, Germany were randomly selected and contacted by personalized letters that included the most relevant eligibility criteria (eg, menopausal status, exercise). A total of 332 women responded by phone or letter and were further assessed for eligibility by phone calls. Eligible women were then invited to bone densitometry and structured interviews to validate their inclusion. We included women with a) early-menopause status defined as 12-60 months amenorrhea, b) osteopenia or osteoporosis without fractures up to a BMD T-Score of $-4 \mathrm{SD}$ at the lumbar spine, femoral neck or total hip. Women with a higher fracture risk (ie, BMD T-Score $>4$ SD) are entitled to pharmaceutic therapy according to German guidelines ${ }^{20}$ and therefore excluded from the study. Women who reported a) medication (ie, Glucocorticoids $>5 \mathrm{mg} / \mathrm{d}$ ), conditions and diseases known to affect bone metabolism or contraindicate group exercise or tests; b) high impact or resistance exercise ( $>45 \mathrm{~min} /$ week) during the last 5 years, ie, exercises with high ground reaction forces like "running"; c) secondary osteoporosis or osteoporotic fractures; d) acute or recent history of cancer (last 5 years); e) regular "high" alcohol consumption (ie, $\geq 60 \mathrm{~g} / \mathrm{d}$ on 5 days/week); f) potential unavailability for more than 6 weeks between January 2019 and September 2020 (ie, during the study period) were excluded (Figure 1). In cases of doubt, the final decisions were made by the study physician. After detailed study information, 21 of the 75 eligible women quit the study. 
women $48-60$ years older were assessed for eligibility: $n=332$

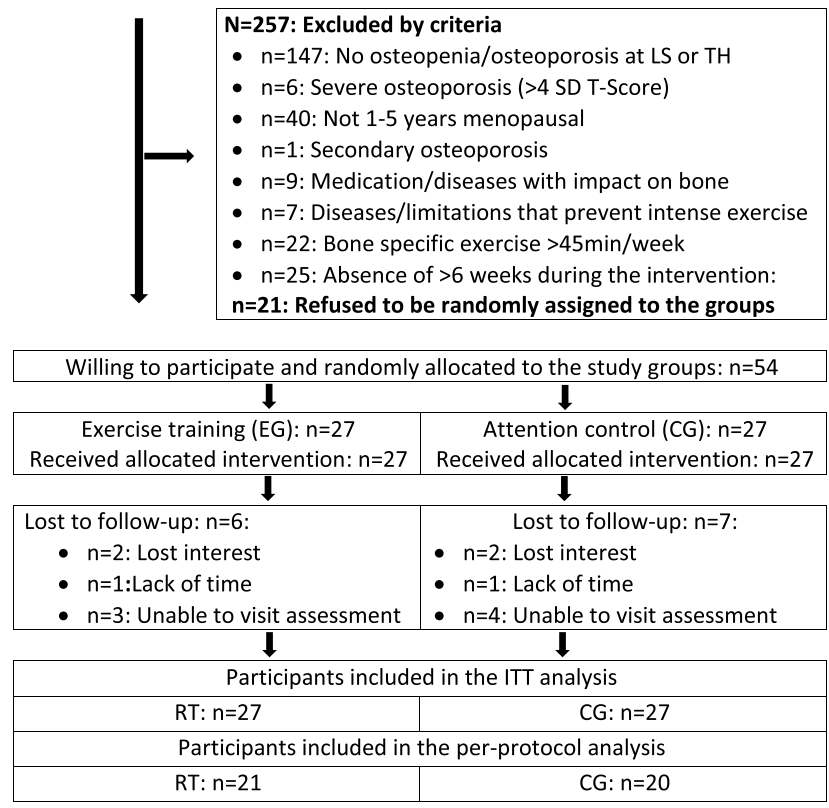

Figure I Participant flow through the study.

Most attributed this to the mandatory randomized group allocation and the inability to join the preferred group. The 54 remaining women eligible and willing to participate were randomly assigned to the groups (Figure 1).

\section{Randomization Procedures}

Using two strata, 54 participants were stratified for baseline lumbar spine BMD and randomly assigned to the $\mathrm{EG}(\mathrm{n}=27)$ or the $\mathrm{CG}(\mathrm{n}=27)$. Participants allocated themselves to the two groups by drawing lots from small opaque capsules ("kinder egg", Ferrero, Italy) placed in a bowl. A researcher not involved in the present project prepared the lots and supervised the randomization procedure. Neither researchers nor participants knew the allocation beforehand ("allocation concealment"). After the randomization procedure, the principal investigator (MH) enrolled participants and instructed them in detail about study specifications.

\section{Blinding}

Outcome assessors and test assistants who were unaware of the participants' group status (EG or CG).

\section{Study Procedure}

ACTLIFE-RCT focused on the effects of exercise on menopausal risk factors and complaints with special regard to BMD. All participants were provided with Cholecalciferol (Vit-D) and Calcium (Ca) supplements in order to realize current recommended intake (ie, $800 \mathrm{IU} / \mathrm{d}$ Vit-D; $1000 \mathrm{mg} / \mathrm{d} \mathrm{Ca},{ }^{20}$ details see below). Participants were asked to maintain their usual dietary intake during the study; further, all women were requested to maintain their lifestyle including habitual physical activity and exercise habits.

\section{Intervention}

\section{Exercise Group}

In summary, our exercise protocol was tailored to the complex risk factor profile of (early) postmenopausal women and used a multimodal approach. We placed particular emphasis on BMD at LS and hip with a mixture of weight-bearing exercise mainly involving aerobic dance with moderate to high ground reaction forces, jumping and resistance exercise. The latter was designed as singleset exercise training (HIT-RT) with high intensity and effort using intensifying strategies. ${ }^{21}$ The intervention was structured in blocks of 10-12 weeks of highintensity/high effort exercise, interspersed with 4-5 weeks of recreational exercise between each phase. During the 10-12 week phases, three supervised group sessions/week (s/w) with 8-10 participants/group were conducted in our lab (Mondays and Wednesday $\approx 40 \mathrm{~min}$ ) and a dedicated women's gym that offered RT (Friday or Saturday $\approx 60 \mathrm{~min}$ ) on devices, while during the recreational phase one supervised session in our lab was replaced by a video-guided individual home session of 15 min involving exercises practiced during the joint sessions. During the first four weeks of exercise, we focused on briefing, familiarization, learning of correct movements and lifting technique, body sensation and the use of the rate of perceived exertion approach.

Aerobic sequence. After 5 min of low-impact aerobic dance for warm-up, participants took part in $15 \mathrm{~min}$ of high impact aerobic dance with 6-10 varying movements of progressively increasing mechanical intensity (ground reaction forces: GRF: 2.0-3.0 body mass; ground reaction forces of different movements were determined in previous studies with comparable cohorts of early postmenopausal women). ${ }^{17,22}$ In summary, 80-120 high impact movements were conducted during each aerobic dance session. After 4 weeks of moderate-intensity continuous exercise, we applied high-intensity interval training (HIIT) with varying cardiovascular exercise intensity. Once a week, 60 seconds of high-intensity phases $(\approx 80-85 \%$ HRmax) were interspersed with 60 seconds of lower intensity ( $\approx 65-70 \%$ HRmax) exercise, the second session 
applied a corresponding 30s/30s exercise protocol. Exercise intensity was checked by regular analysis of the heart rate monitors provided to the participants. HRmax was determined by a stepwise treadmill test to a voluntary maximum, using steps of $3 \mathrm{~min}$, speed increases of $1 \mathrm{~km} / \mathrm{h}$ and $1 \%$ incline of the treadmill. Apart from high exercise intensity and proper movements, emphasis during the aerobic dance session was on enjoyment and interaction of the participants rather than complicated choreographies.

After 6 months, we introduced a dedicated jumping sequence. We applied a jumping course with 7 step boards of varying height that participants jumped over or on. This jump sequence was repeated 8 times with 20 s of rest between each repetition. Step-boards were progressively elevated (from 20 to $36 \mathrm{~cm}$ ) over the course of the intervention. Peak GRF for the deep jump from the highest step board averaged at about $4-4.5 \mathrm{x}$ body mass.

Dynamic resistance exercise training (DRT) can be considered a key component of our exercise protocol. Participants were provided with detailed training logs that prescribed exercises, number of repetitions (gym) or loading duration (circuit), movement velocity and absolute exercise intensity (or "effort").

After the initial 4-week conditioning phase, we introduced the periodized DRT approach and applied three (intensity-based) linearly periodized 4-week phases, with every fourth week as a recovery week with low effort. During the DRT sequence conducted in our lab, a singleset approach that addressed all the main muscle groups (calf rises, lunges, leg-press, half squat, (half) squats; back extension (roman chair), deadlifts; single side lateral rows, trapezius and latissimus pulldowns, bench dips, incline dumbbell bench press) was applied in a circuit mode. Correspondingly, we did not prescribe the number of reps but the duration of the loading cycles, which varied between $40 \mathrm{~s}, 60 \mathrm{~s}$ and $80 \mathrm{~s}$ and the time under tension per repetition (TUT: $2 \mathrm{~s}$ (concentric) - $1 \mathrm{~s}$ (isometric) $2 \mathrm{~s}$ (eccentric), $=2-1-2$ ). We consistently applied 30s of rest between the exercises. Thus, the number of repetitions (reps) averaged between 8 (40s) and 16 reps (80s). Exercise intensity during the first 12 -week block was prescribed as repetition maximum minus 1-2 reps. ${ }^{23,24}$ During the second 12-week (phase 2) high-intensity DRT period, we manipulated movement velocity. Applying a TUT that varied between explosive movement (except back extension and deadlift) - 1s (isometric) 1s/(isometric) $-2 \mathrm{~s}$ (eccentric) and $4 \mathrm{~s}-0 \mathrm{~s}-4 \mathrm{~s}$, the number of repetitions (reps) ranged between 5 and 20 reps per session. Similar to Phase 1, exercise intensity was prescribed using the repetition in reserve (RIR) approach of Zourdos et al, ${ }^{24}$ and the set endpoint definition of Steele et $\mathrm{al}^{23}$ (nRM; repetition maximum minus 1-2 reps). After 32 weeks of exercise (phase 3), we introduced the repetition maximum approach. ${ }^{23}$ Set endpoint was when trainees completed the final repetition possible, but if the next repetition was attempted, they would definitely achieve muscular failure (ie, inability to conduct the rep). Comparable to the previous training period movement velocity and the number of reps/loading cycle was flexibly manipulated; relative intensity varied from $60 \% 1 \mathrm{RM}$ for 20 reps $(80$ s; $2-0-2)$ and $80 \% 1$ RM for 6 reps (30s; expl. $1 \mathrm{~s}-3 \mathrm{~s})$. Of importance, sets conducted with explosive movements were consistently nRM sets. After 4 weeks of low effort exercise, in Phase 4 we introduced the dropset approach, typical for HIT-RT. We manipulated the order of exercises within the circle to address either the same/related muscle groups (eg, lunges-leg press-half squat) or agonist and antagonist (eg, leg press-deadlift) in a row. Two or three exercises were included in a superset sequence. Intermitted by 5 weeks of low effort exercise, we conducted the present follow-up assessment and aimed to start with Phase 5 in March 2020. Phase 5 should focus on work to failure, simplified the inability to (properly) conduct the concentric phase of the current rep. ${ }^{23}$ However, due to COVID-19 lockdown, we had to stop exercising and after 2 months of obligatory break we finally stopped the study initially designed for 18 months.

Apart from circuit training in our lab, participants trained on dedicated resistance training machines in a women's gym once a week (Fridays or Saturdays). In general, the exercise training on devices was structured much the same way, the only difference being that the number of reps rather than the time of the loading cycle (circuit) was specified. Women completed a 15-minute warm-up on a cross-trainer (65-70\% HRmax), before starting the DRT. The supervised single-set exercise approach of the gym training addressed 13-15 exercises (leg press, -extension, -curls, -adduction, -abduction, latissimus front pulleys, rowing, roman chair, trunk extension, -flexion, inverse fly, bench press, military press, lateral raises, shoulder/triceps press). Similar to the circuit training, we scheduled a linearly periodized exercise protocol with a varying number of repetitions, movement velocity, and varying intensity (nRM to RM; 5-20 reps at nRM -1 rep corresponding to $65-82.5 \%$ $1 \mathrm{RM}^{25}$ ) with a rest between the sets averaging $60 \mathrm{~s}-120$ 
s. Relative peak intensity (eg, 4 reps to $\mathrm{RM} \approx 85 \% 1 \mathrm{RM}$ ) was slightly higher in the gym protocol. The same intensifying strategies were otherwise applied.

During the 4-5 week low effort periods, one circuit session (see above), one 45 min session of stretching and floor exercises (see control group) and one video-guided home training session (see control group) of 15 min were conducted.

\section{Control Group}

The exercise in attention control group focused on stability, flexibility and well-being, albeit with a strong emphasis on applying an exercise protocol that should not relevantly affect "bone", "body composition" or "maximum strength/power". During the 13-month intervention period, we completed 2 cycles of 12 weeks of supervised group exercises (45 min) intermitted by 12-14 weeks of non-supervised, video-guided home exercise (15 min).

The consistently supervised group training ( $8-10$ participants/group) session started with 15 minutes of walking/ marching exercise followed by 20 minutes of stretching and easy floor exercises and 10 minutes of cool down. Lower and upper calf, hamstring, thigh, gluteal, hip flexors, lower and upper back, abdominal, and pectoralis sites were exercised with one set of stretching routines each with $30 \mathrm{~s} /$ exercise of moderate intensity. Participants were asked not to exceed a pleasant feeling of tension. Predominately easy isometric floor exercises in a sitting, supine or prone position focused predominately on trunk muscle groups. Two sets each of 6-8 varying exercises/session with $10 \mathrm{~s}$ of moderate intensity ("5" on Borg CR 10) ${ }^{26}$ and $30 \mathrm{~s}$ of rest were conducted. During the 10 minutes of cool down, the instructor presented different "fantasy journeys" to encourage general relaxation or body awareness.

During the non-supervised phases, participants were provided with training videos that summarized the joint training session. Fifteen minutes of stretching and isometric exercises, which had been demonstrated during the supervised training period, were conducted during the home training session. The non-supervised training period finished immediately before the 13-month follow-up assessment.

\section{Vitamin-D and Calcium Supplementation}

All participants were requested to take two capsules of cholecalciferol (MYPROTEIN, Cheshire, UK) of $2500 \mathrm{IE} /$ $\mathrm{d}$ once a week (ie, $5000 \mathrm{IE} /$ week) independently of their baseline $25 \mathrm{OH} \mathrm{D}$ levels. We aimed to ensure a calcium intake of $1000 \mathrm{mg} / \mathrm{d}$ for all the participants. ${ }^{20}$ Dietary calcium intake was determined using dietary calcium questionnaires (Rheumaliga, Switzerland); to determine the dose of calcium provided via calcium carbonate capsules (Sankt Bernhard, Bad Dietzenbach, Germany).

\section{Compliance with the Exercise Intervention}

Participant attendance was recorded by the instructor and verified by the gym's chip card system. Adherence to the exercise protocol, particularly the application of proper exercise intensity was checked by a) reviewing the training logs after each 12 -week mesocycle and b) by the instructors that monitor the load/repetition proportion during the sessions.

\section{Study Outcomes \\ Primary Study Outcome(s)}

- Bone Mineral Density changes at the lumbar spine and hip as determined by Dual-Energy X-Ray Absorptiometry (DXA) from baseline to 13-month follow-up assessment.

\section{Secondary Study Outcomes}

- Soft lean body mass, and total and abdominal body fat changes as determined by DXA from baseline to 13-month follow-up.

- Changes of cardiometabolic risk as determined by the metabolic syndrome ${ }^{27} \mathrm{Z}$-Score ${ }^{28}$ from baseline to 13 month FU.

- Changes of menopausal symptoms as determined by menopausal rating scale $\mathrm{II}^{29}$ from baseline to 13month FU.

- Maximum dynamic hip/leg extension strength changes as determined by an isokinetic leg press from baseline to 13-month FU.

- Maximum jumping height as determined by a force plate from baseline to 13-month FU.

\section{Changes of Trial Outcomes After Trial Commencement}

Due to the COVID-19 lockdown in Bavaria, which included the closing of all sports and exercise facilities (17 March to 8 June 2020), we decided after 8 weeks of deconditioning to discontinue the study. We were unable to conduct the MRI assessment initially planned after 18 months at the new 13-month endpoint. 


\section{Assessments}

High emphasis was placed on standardized testing and assessments. Participants were asked to avoid changes in dietary intake and physical activity prior to the tests. All the tests/assessments were conducted at the same time of day ( $\pm 90 \mathrm{~min})$, at the same location, using the same calibrated devices, but not always (ie, blood sampling, strength/power assessments) by the same test assistant.

Body height was determined using a Holtain stadiometer (Crymych Dyfed., Great Britain), body mass was assessed using the scale function of the direct-segmental, multi-frequency Bio-Impedance-Analysis (DSM-BIA; InBody 770, Seoul, Korea). Total and regional body composition and areal BMD at the lumbar spine (LS) between L1-L4 vertebrae and total hip (TH) were assessed by DXA (QDR 4500a, Discovery-upgrade, Hologic Inc., Bedford, USA). Abdominal body fat was segmented between the lower end of the 12th thoracic vertebra and the upper end of the iliac crest. Segmentation of LS and TH area and regional body composition (ie, abdominal region, thigh region) was conducted using the "compare mode", so that baseline area and placement of the baseline assessment could be reproduced exactly during the FU assessments.

Blood was drawn from an antecubital vein after an overnight fast between 7:00 and 9:00 on a different day from the DXA, blood pressure or functional assessments. Serum samples were centrifuged for $20 \mathrm{~min}$ at $3000 \mathrm{RPM}$; glucose, total cholesterol, HDL and LDL cholesterol and triglycerides (Olympus Diagnostica GmbH, Hamburg, Germany) were immediately analyzed in the "Zentrallabor" of the Medical Department, FAU.

Immediately after $10 \mathrm{~min}$ of rest (during DXA assessment), blood pressure (RR) was evaluated in a lying position with an automatic oscillometric device (Bosco, Bosch, Jungingen, Germany). Subjects were requested to arrive at the assessment in a relaxed, non-fasting condition but having refrained from coffee or tea for at least 2 hours prior to testing. We calculated Mean Arterial Pressure (MAP) (diastolic RR + diastolic RR + systolic RR)/3.

We calculated the MetS-Z-Score using the International Diabetes Federation definition ${ }^{27}$ and applying the approach of Johnson et $\mathrm{al}^{28}$ for the calculation. We calculated the MetS-Z-Score as: [(50 - HDL-cholesterol) /SD HDL-C $]+[($ triglycerides -150$) / \mathrm{SD}$ TriGly $]+$ $[($ Glucose -100$) /$ SD Glucose $]+[($ waist circumference 80)/SD WC $]+[(\mathrm{MAP}-100) / \mathrm{SD}$ MAP $]$.
We used an isokinetic leg press (CON-TREX LP, Physiomed, Laipersdorf, Germany) to determine maximum isokinetic leg/hip extensor strength. We conducted the test in a sitting, slightly supine position, hip and chest fixed with straps. The participants' feet were positioned on a flexible sliding footplate and also fixed with straps. The range of motion was $30^{\circ}$ to $90^{\circ}$ within the knee angle; velocity of the movement was $0.2 \mathrm{~m} / \mathrm{s}$. Emphasis was placed on the concentric part of the movement (ie, hip/ leg extension). After familiarization with the testing procedure, the women conducted five reps with maximum effort ("push as strongly as possible"). Two minutes of rest were specified between the two trials. The higher value of both trials was included in the data analysis.

We determined lower extremity power by a countermovement jump (CMJ) with hands on hips (ie, no arm swing) during the trial. We asked participants to "jump as high as possible" starting from an upright position. Countermovement depth was not specified. We asked participants to maintain extension in the hip, knee, and ankle joints to prevent any additional flight time by bending their legs. Jumping height (present outcome) and power were determined by a force platform (KMP Newton GmbH, Stein, Germany). The jumping height was calculated automatically by the software provided by the manufacturer based on ground reaction forces.

All participants had to complete a standardized baseline questionnaire ${ }^{17}$ that asked for a) demographic parameters; b) diseases, physical limitations and pharmacologic therapy under special consideration of osteoporosis risk and ability to frequently conduct intensive exercise; c) dietary supplements; d) pain frequency and severity at the lumbar spine region; e) lifestyle, with high emphasis placed on physical activity and exercise; ${ }^{30}$ and f) menopausal complaints as per the Menopause Rating Scale (MRS II) provided by Hauser et al. ${ }^{29,31}$ After 7 and 13 months, all participants had to complete a follow-up (FU) questionnaire that particularly focused on changes in parameters (ie, pharmacologic therapy, diseases, operations, lifestyle, diet, exercise) that might have affected the present study outcomes. Strong emphasis was placed on checking questionnaires for consistency, completeness and accuracy together with the participants.

Four-day diet records were completed at baseline and after 7- and 13-month FU. We briefed and instructed participants in detail on how to keep the diet records (Freiburger Nutrition Record (nutri-science, Hausach, Germany)). We asked participants to protocol 3 weekdays and one weekend day 
representative for their nutritional habits. Diet records were carefully analyzed consistently by the same researcher. In cases of unlikely results (eg, energy intake $<1000 \mathrm{kcal} / \mathrm{d}$ or $>3500 \mathrm{kcal} / \mathrm{d}$ ) the results were discussed with the participants. In all cases, the women were requested to provide another diet record based on more representative days.

\section{Sample Size Calculation}

The sample size calculation was based on the primary study outcome "BMD changes at the LS" after 18 months. We assumed an effect ( $\Delta$-EG vs $\Delta-C G$ ) on BMD-LS of 2.0 $\pm 2.5 \%$ as determined in other exercise studies with early postmenopausal women in our $1 \mathrm{lab}^{22,32}$ and applied aindependent $t$-test based sample size calculation. In summary, the sample size required to generate $80 \%$ power $(1-\beta)$ and alpha $=0.05$ was 25 participants per group. We included 27 participants to allow for drop-outs within an additional per-protocol analysis for the primary study outcome.

\section{Statistical Analysis}

As prescribed for an RCT, we conducted an intention to treat (ITT) analysis that included all participants assigned to the two study arms (EG vs CG) at baseline. Additionally, a perprotocol analysis was performed for the primary study outcome that only included participants with complete datasets independently of compliance with the exercise protocol or potentially confounding factors. Using $\mathrm{R}$ statistics software, ${ }^{33}$ we calculated multiple imputations (ITT) in combination with Amelia II. ${ }^{34}$ The full data set was used for multiple imputations with imputation being repeated 100 times. Imputation diagnostic plots provided by Amelia II indicated that imputation for primary and secondary outcomes worked well. Normal distribution of the study endpoints was checked by statistical (Shapiro-Wilks) and graphical (qq-plots) procedures. Within-group changes were tested for by dependent $t$-tests. Pairwise $t$-test comparisons ( $\mathrm{EG}$ vs $\mathrm{CG}$ ) with pooled $\mathrm{SD}$ adjusted for baseline values were applied to test for group differences. Alternatively, a repeated measures ANOVA (group by time interaction) was calculated within the per-protocol analysis. We applied 2-tailed tests; significance was accepted at $\mathrm{p}<0.05$. Standardized mean difference (SMD) was calculated according to Cohen (Cohen's d). ${ }^{35}$

\section{Results}

Baseline characteristics of the ACTLIFE-RCT study are shown displayed in Table 1. Of note, dietary protein intake was high in both groups. Albeit non-significant, $25 \mathrm{OHD}$
Table I Baseline Characteristics of the ACTLIFE-RCT Study

\begin{tabular}{|l|c|c|}
\hline Variables & $\begin{array}{c}\text { CG (n=27) } \\
\text { MV } \pm \text { SD }\end{array}$ & $\begin{array}{c}\text { EG (n=27) } \\
\text { MV } \pm \text { SD }\end{array}$ \\
\hline Age [years] & $54.5 \pm 1.6$ & $53.6 \pm 2.0$ \\
Body height [cm] & $164.5 \pm 8.2$ & $164.2 \pm 6.0$ \\
Body mass [kg] & $67.4 \pm 14.6$ & $64.0 \pm 9.6$ \\
BMI [Weight/(Height*Height)] & $24.9 \pm 4.8$ & $23.7 \pm 3.4$ \\
Calcium intake [mg/d] & $642 \pm 265$ & $645 \pm 252$ \\
Vit-D level (25-OHD) [ng/mL] & $21.6 \pm 10.8$ & $27.8 \pm 11.7$ \\
Years after menopause [yr] & $3.5 \pm 1.1$ & $3.7 \pm 1.0$ \\
Exercise volume [min/week] & $45.6 \pm 38.4$ & $63.7 \pm 47.5$ \\
Waist circumference [cm] & $91.1 \pm 9.9$ & $87.8 \pm 8.6$ \\
Energy intake ${ }^{\mathrm{a}}[\mathrm{kcal} / \mathrm{d}]$ & $2067 \pm 355$ & $2009 \pm 444$ \\
Protein intake [g/kg/body mass/d] & $1.20 \pm 0.21$ & $1.18 \pm 0.27$ \\
Fat intake [g/d] & $86 \pm 24$ & $84 \pm 21$ \\
Alcohol intake ${ }^{\mathrm{a}}[\mathrm{g} / \mathrm{d}]$ & $5.53 \pm 6.39$ & $2.63 \pm 4.06$ \\
Smokers [\%] & $\mathrm{II}$ & 7 \\
Ovariectomy <50 years [n] & 0 & $\mathrm{I}^{\mathrm{b}}$ \\
Family disposition ${ }^{\mathrm{c}}[\mathrm{n}$ ] & 9 & 7 \\
\hline
\end{tabular}

Notes: ${ }^{2}$ As determined by a 4-day dietary protocol, see methods; ${ }^{\text {b At age }} 47$ years; ${ }^{c}$ Fragility fractures or verified osteoporosis in close relatives (parents, aunts, uncles, grandparents).

concentration and alcohol intake varied considerably between the groups. Dietary calcium intake was similar (low) in both groups (Table 1); however in contrast to cholecalciferol supplementation (800-1000 IE/d; $n=8$ in EG and CG, respectively), no women used calcium supplements before the study start. Nevertheless, baseline Vit-D levels were on average below recent recommendations. ${ }^{19}$ Further, dietary protein intake was quite high in both groups. As intended, exercise volume was low in both groups and focused predominately on aerobic exercise.

Six women in the EG and seven women of the CG were lost to a 13-month follow-up. Three women in each group quit the intervention (Figure 1). Four in the CG and three women in the EG were unable to visit the 13-month follow-up assessment, which had a very close window of only 3 days due to the rapid and restrictive COVID-19 lockdown procedure in Bavaria. Reasons for withdrawal are listed in Figure 1.

On average, participants in the EG completed $129 \pm 21$ of 164 possible sessions $(79 \pm 12 \%)$. Of note, the session in our lab was more popular than the gym sessions (83\% vs $75 \%$ ). The attendance rate of the CG for the two supervised and two 12-week non-supervised exercise training periods averaged $78 \pm 14 \%$. Participants reported that they consistently adhered to the exercise protocol; however, monitoring of "effort" by checking the relationship of reps and load selected to realize $\mathrm{RM}$ indicated that 
Table 2 Baseline Data and Changes of BMD at LS and TH in the CG and EG and Corresponding Between Group Differences. *P $<0.05$

\begin{tabular}{|c|c|c|c|c|}
\hline & CG MV $\pm S D$ & EG MV $\mathbf{S D}$ & Difference MV (95\% Cl) & p-value \\
\hline Bone Mineral Density (BMD) at the lumbar spine $\left[\mathrm{mg} / \mathrm{cm}^{2}\right]$ & & & & \\
\hline Baseline & $0.904 \pm 0.097$ & $0.873 \pm 0.130$ & $\longrightarrow$ & 0.314 \\
\hline Changes & $-.009 \pm 0.018^{*}$ & $0.002 \pm 0.018$ & 0.011 ( 0.001 to 0.021$)$ & 0.027 \\
\hline \multicolumn{5}{|l|}{ Bone Mineral Density (BMD) at the total hip $\left[\left[\mathrm{mg} / \mathrm{cm}^{2}\right]\right.$} \\
\hline Baseline & $0.826 \pm 0.056$ & $0.804 \pm 0.099$ & $\longrightarrow$ & 0.337 \\
\hline Changes & $-.009 \pm 0.020 *$ & $-.001 \pm 0.016$ & $0.008(-.002$ to 0.017$)$ & 0.129 \\
\hline
\end{tabular}

women did not always follow the RM specification. This is particularly the case for the first high-intensity phase.

\section{Primary and Secondary Study Outcomes}

LS-BMD, the primary study outcome, was maintained in the $\mathrm{EG}(\mathrm{p}=0.517)$ and decreased significantly in the $\mathrm{CG}(\mathrm{p}=0.015)$ (Table 2). LS-BMD changes differ significantly between the two groups ( $\mathrm{p}=0.026 ; \mathrm{SMD}=0.62$; Table 2 ). In parallel, BMD at the total hip decreased significantly in the $\mathrm{CG}(\mathrm{p}=0.023)$ and was maintained in the $E G(p=0.756)$. Corresponding differences between the groups were non-significant $(p=0.129$, $\mathrm{SMD}=0.38$; Table 2). Thus, we reject our primary hypothesis that the EG demonstrated greater effects on BMD at the LS and total hip compared with an attention CG.

LBM increased in the EG $(\mathrm{p}<0.013)$ and was maintained in the $C G(p=0.127)$. Differences between the group changes were significant $(\mathrm{p}<0.026$, SMD: 0.63 ; Table 3).

Total body fat percentage decreased in the EG $(p<0.001)$ and was maintained in the CG $(p=0.930)$. Changes between EG and CG for total body fat percentage were significantly different $(\mathrm{p}<0.002$; SMD: 0.93; Table 3). In parallel, abdominal body fat percentage was maintained in the CG $(\mathrm{p}=0.835)$ but decreased $(\mathrm{p}=0.002)$ in the EG Changes among the EG and CG varied between the two groups ( $\mathrm{p}=0.011$, SMD: 0.72; Table 3).

The MetS-Z-Score was maintained in the EG $(\mathrm{p}=0.804)$ and worsened $(\mathrm{p}=0.041)$ in the CG. However, pre- to postintervention changes in MetS-Z-score did not differ between EG vs CG (Table 4). Favorable effects of exercise were determined for waist circumference (EG: $-2.9 \pm 3.9 \mathrm{~cm}$ vs CG: $-0.4 \pm 3.6 \mathrm{~cm}, \mathrm{p}=0.010$; SMD: 0.75) and MAP (EG: 1.7

Table 3 Baseline Data and Changes of Anthropometric Parameters in the CG and EG and Corresponding Between Group Differences. $* \mathrm{P}<0.05 ; * * \mathrm{P}<0.01 ; * * * \mathrm{P}<0.001$

\begin{tabular}{|c|c|c|c|c|}
\hline & CG MV $\pm S D$ & EG MVISD & Difference $\mathrm{MV}(95 \% \mathrm{Cl})$ & p-value \\
\hline \multicolumn{5}{|c|}{ (Soft) Lean Body Mass (LBM)[kg] } \\
\hline Baseline & $41.34 \pm 6.43$ & $40.39 \pm 4.78$ & $\longrightarrow$ & 0.544 \\
\hline Changes & $-0.37 \pm 1.34$ & $0.39 \pm 1.08^{*}$ & $0.76(0.10$ to 1.43$)$ & 0.026 \\
\hline \multicolumn{5}{|c|}{ Total Body Fat Percentage [\%] } \\
\hline Baseline & $34.2 \pm 6.9$ & $34.0 \pm 5.0$ & - & 0.866 \\
\hline Changes & $-0.02 \pm 1.55$ & $-1.44 \pm 1.49 * * *$ & $1.42(0.54$ to 2.29$)$ & 0.002 \\
\hline \multicolumn{5}{|c|}{ Abdominal Body Fat Percentage [\%] } \\
\hline Baseline & $28.6 \pm 9.2$ & $28.5 \pm 7.0$ & $\longrightarrow$ & 0.972 \\
\hline Changes & $0.08 \pm 2.02$ & $-1.50 \pm 2.33^{* *}$ & $1.58(0.38$ to 2.78$)$ & 0.011 \\
\hline
\end{tabular}

Table 4 Baseline Data and Changes of the Metabolic Syndrome Z-Score in the CG and EG and Corresponding Between Group Differences

\begin{tabular}{|l|c|c|c|c|}
\hline & CG MVISD & EG MV $\mathbf{S D}$ & Difference MV (95\% CI) & p-value \\
\hline Metabolic Syndrome Z-Score [Index] & & & & \\
$\quad$ Baseline & $-4.15 \pm 3.99$ & $-5.51 \pm 2.43$ & - & 0.143 \\
Changes & $0.77 \pm 2.07$ & $0.08 \pm 1.29$ & $0.69(-0.20$ to 1.65$)$ & 0.149 \\
\hline
\end{tabular}


Table 5 Baseline Data and Changes of Menopausal Symptoms According to MRS II and Low Back Frequency in the CG and EG and Corresponding Between Group Differences. ${ }^{* *} \mathrm{P}<0.01$

\begin{tabular}{|l|c|c|c|c|}
\hline & CG MV \pm SD & EG MV \pm SD & Difference MV (95\% Cl) & P-value \\
\hline $\begin{array}{l}\text { Menopause Rating Scale II [Score Points] }{ }^{\mathbf{a}} \\
\text { Baseline }\end{array}$ & $1.20 \pm 0.52$ & $1.06 \pm 0.64$ & -0.365 \\
Changes & $-0.01 \pm 0.54$ & $-0.32 \pm 0.5 I^{* *}$ & $-0.31(0.03$ to 0.59$)$ & 0.029 \\
\hline
\end{tabular}

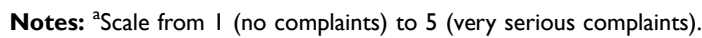

\pm 8.5 vs $\mathrm{CG}:-2.8 \pm 6.0 \mathrm{mmHg}, \mathrm{p}=0.030 \mathrm{SMD}$ : 0.61 ) only, while changes in fasting blood glucose (EG: $4.4 \pm 8.2$ vs CG: $4.2 \pm 8.3 \mathrm{mg} / \mathrm{dl}, \mathrm{p}=0.933$, SMD: 0.02), triglycerides (EG: $12.2 \pm 40.6$ vs CG: $11.1 \pm 29.3 \mathrm{mg} / \mathrm{dl}, \mathrm{p}=0.907$, SMD: 0.03 ) and HDL-C (EG: $1.0 \pm 8.5$ vs CG: $4.1 \pm 8.6 \mathrm{mg} / \mathrm{dl}, \mathrm{p}=0.187$, SMD: 0.36) did not differ between EG and CG.

Menopausal symptoms as determined by the MRS II (Table 5) improved in both groups; however, the changes from pre- to post-intervention were only significant in the EG (EG: $p=0.002$ vs CG: $p=0.891$ ). Further, significant between-group differences for MRS were observed $(\mathrm{p}=0.029$; SMD: 0.59). However, although changes of all the subscales ("dimensions") of the MRS II, ie, somato-vegetative, psychological, and urogenital complexes, were more favorable in the $\mathrm{EG}$, significant differences between EG and CG ( $\mathrm{p} \geq .10)$ were not observed.

Finally, maximum leg press $(\mathrm{p}<0.001)$ and jumping performance $(\mathrm{p}<0.001)$ increased in the EG and improved only slightly (strength: $\mathrm{p}=0.314$; power: $\mathrm{p}=0.197$ ) in the CG (Table 6). Differences between the groups were significant for maximum hip/leg extension strength $(\mathrm{p}<0.001$; SMD: 2.33) and lower extremity power $(p=0.001$; SMD: 1.66).

Thus, apart from hypothesis (d) (ie, effects on cardiometabolic risk), all the hypotheses addressed were confirmed. In each case, the per-protocol analysis confirmed the result of the ITT analysis.
We did not observe changes or between-groups differences for dietary intake parameters (ie, energy, carbohydrate, fat, protein, alcohol intake), lifestyle including physical activity/exercise or pharmacologic therapy that might have affected our result. In parallel, no changes in diseases with impact on our study outcome or absence $>2$ weeks from exercise were reported. 25 OHD serum concentration improved significantly and comparably (EG: 9.3 \pm 9.1 vs $\mathrm{CG}$ : $9.1 \pm 10.5 \mathrm{ng} / \mathrm{mL}$ ) in both groups.

\section{Discussion}

In this study, we aimed to determine the effect of a multipurpose exercise program on menopausal risk factors and complaints in early postmenopausal women with osteopenia and osteoporosis. ${ }^{36}$ Our training protocol was based on international recommendations, ${ }^{37-39}$ metaanalyses $^{16}$ and a review of "European experience" (limited to ACTLIFE partner countries) on secondary and tertiary osteoporosis prevention by means of exercise. However, in order to generate a multipurpose exercise protocol, we included types of exercise (eg, endurance exercise) that also focus on other risk factors related to the menopausal transition. Despite the sophisticated exercise protocol, BMD at the total hip showed no change as a result of the intervention. By comparison, Watson et al, ${ }^{40}$ who used a much more condensed (2x $30 \mathrm{~min} / \mathrm{w}$ ) high-intensity resistance and impact exercise protocol reported a much higher effect on BMD at LS (EG: $2.9 \pm 2.8 \%$ vs CG: -1.2

Table 6 Baseline Data and Changes of Maximum Hip/Leg Extension Strength and Maximum Jumping Height in the CG and EG and Corresponding Between Group Differences. *** $\mathrm{P}<0.001$

\begin{tabular}{|c|c|c|c|c|}
\hline & CG MV \pm SD & EG MV \pm SD & Difference $M V(95 \% \mathrm{Cl})$ & p-value \\
\hline \multicolumn{5}{|l|}{ Maximum hip/leg extension strength (leg press) [N] } \\
\hline Baseline & $2056 \pm 576$ & $2073 \pm 429$ & $\longrightarrow$ & 0.901 \\
\hline Changes & $46 \pm 187$ & $607 \pm 285 * * *$ & 561 (427 to 694) & $<0.001$ \\
\hline \multicolumn{5}{|l|}{ Maximum jumping height (counter movement jump) $[\mathrm{cm}]$} \\
\hline Baseline & $19.1 \pm 3.2$ & $19.4 \pm 3.7$ & $\longrightarrow$ & 0.807 \\
\hline Changes & $0.55 \pm 1.82$ & $4.10 \pm 2.41 * * *$ & 3.56 (2.38 to 4.72$)$ & $<0.001$ \\
\hline
\end{tabular}


$\pm 2.8 \%, \quad \mathrm{p}<0.001)$ and $\mathrm{FN} \quad(0.3 \pm 2.6 \%$ vs $-1.9 \pm 2.6 \%$, $\mathrm{p}=0.004)$ in their albeit older $(65 \pm 5 \mathrm{yr})$ cohort of women with osteoporosis. One may argue that the early-postmenopausal status of our participants might have contributed to this difference; however, a recent meta-analysis16 indicates a somewhat higher (... but non-significant) plausible explanation for the rather low effects on BMD might be the careful gradual progression of the exercise intensity in the present intervention. This gradual progression might not have reached a threshold for bone adaption until 5-6 months. With the remaining 7 months of exposure to "effective" exercise too short to elicit the full amount of mineralized bone, ${ }^{41}$ assuming bone adaptation in adults is triggered predominately by remodeling. ${ }^{42,43}$ Although the loading intensity triggered by HIT-DRT (up to $\approx 80 \% 1 \mathrm{RM}$; corresponding to 5 reps with $\mathrm{nRM}$ ) and high impact aerobic dance (up to GRF 3.0 body mass) applied after the short conditioning should have stimulated bone metabolism in predominately untrained females, the aforementioned highly effective 8-month study of Watson $^{40}$ suggests otherwise. Finally, the "exerciseinduced" effects in the attention control group may have confounded our result. However, one session of 45 or 15 min, respectively, of easy floor exercises, flexibility and wellbeing should not relevantly affect BMD changes (Table 2) in the CG. ${ }^{44,45}$ Indeed, LS and TH-BMD developments of the $\mathrm{CG}$ were inconspicuous considering the average menopausal age $(3.7 \pm 1.0 \mathrm{yrs})$ in this group. In this context, it should be considered as a study limitation that we applied a rather broad window of 5 years for the "early postmenopause". However, the most prominent bone decline might occur during the 3 -year phase of the transmenopause, ie, one year before and two years after the final menstrual period. ${ }^{36}$

Apart from bone, LBM and body fat are also negatively impacted by the menopausal transition. ${ }^{46,47}$ We found significant positive effects on both parameters. However, the effect on LBM was of a much smaller magnitude $(0.76 ; 95 \% \mathrm{CI}: 0.10$ to $1.43 \mathrm{~kg})$ than described in provided by a recent meta-analysis ${ }^{48}$ of younger $(\leq 50$ years) women $(1.45,0.4-3.3 \mathrm{~kg})$. Nevertheless, applying a similar exercise protocol (EG: 3x 45-60 min/week, intense RT and high impact exercise for 12 months vs CG: low intensity exercise) in our earlier TRACE study on early postmenopausal (1-3 years post) women ${ }^{18,22}$ revealed comparable results $(0.54,0.13$ to $0.94 \mathrm{~kg})$. We observed an ongoing LBM effect with a $\approx 50 \%$ higher LBM effect after 13 months compared with 7-month FU
$(0.52,0.17-0.88 \mathrm{~kg})$. Effects on total and abdominal body fat were high (SMD: 0.72 and 0.92). Total body mass decreased significantly by about $-4 \%$ (or $-1.15 \pm 1.23 \mathrm{~kg}$ ) in the EG and was maintained in the CG. A parallel, slightly less prominent development was observed for abdominal body fat percentage (Table 3 ). In general, aerobic exercise (AE) is considered more effective for decreasing body fat compared with RT. ${ }^{49}$ However, the TRACE study, ${ }^{18,22}$ which applied a $\approx 50 \%$ higher AE volume (with similar exercise intensity up to $80-85 \%$ HFmax) compared to ACTLIFE, was unable to demonstrate effects on total and abdominal body fat mass $(\mathrm{p} \geq .703, \mathrm{SMD}<0.2)$. Of note, unlike LBM, total and abdominal body fat did not decrease after 7 months. This is surprising, since the literature attributes a considerable proportion of fat reduction to increases in resting metabolic rate induced by muscle mass. ${ }^{50,51}$ ACTLIFE-RCT did not determine significant effects on the MetS-Z-Score (Table 4). Considering the positive effects on body composition and their recognized association to cardiometabolic risk factors $^{52-54}$ expectations of positive effects on MetS$Z$-Score were justified. Few exercise studies have used the MetS- $Z$ as a study outcome, even fewer focus on mixed aerobic/resistance type or dedicated resistancetype exercise. ${ }^{55-63}$ While Bateman et $\mathrm{al}^{55}$ and Earnest et $\mathrm{al}^{58}$ did not report positive effects of a 4- or 9-month DRT in people with diabetes mellitus type II or overweight, sedentary people, other studies that applied 3-4 months of $\mathrm{RT}^{56,60-62}$ or mixed AE/RT protocol ${ }^{57,59}$ have verified significant positive effects on the MetS-Z-Score in male ${ }^{60,61}$ and among predominately postmenopausal female cohorts. ${ }^{56,57,59,62}$ The results largely reflect our 4-month study results, even though effort (RM) and intensity (up to $85 \% 1 \mathrm{RM}$ ) of the exercise protocol were progressively increased.

We determined significant positive effects on menopausal symptoms as determined by the MRS II scale. ${ }^{29}$ However, we did not observe significant exercise effects in the three underlying MRS II components, ie, the somatovegetative, psychological and urogenital dimensions. Previous studies reported positive exercise effects on psychological factors ${ }^{64,65}$ and conflicting effects on vasomotor symptoms. ${ }^{66,67}$ This inconsistency in findings may be due to which MRS II components were included under "somato-vegetative dimension".

Improvements in maximum hip/leg extensor strength and power (ie, maximum jumping height) averaged $27 \%$ (95\%-CI: $19-36 \%)$ and 18\% (95\%-CI: 12 to $23 \%$ ) from 
the 8 -month to the 13 -month FU $(\mathrm{p}<0.05)$ in the EG indicate the effectiveness of our progressive training program. Nevertheless, maximum strength changes were lower compared with other DRT trials in comparable female cohorts $(30-85 \%){ }^{68-73}$ It is likely that this difference is due to the high baseline values in our cohort and slight improvements in the attention CG.

Due to the unintended termination of the study, some features and limitations of ACTLIFE-RCT have to be addressed. 1) The intended 18-month duration meant that we progressed gradually placing emphasis to a stepwise progression of exercise intensity/effort. However, the premature study termination led to the most intense (... and speculative "effective") part of the protocol, ie, the last 6 months, being truncated. Although when applying a strictly progressive protocol, one may argue that the majority of the physiological changes occurred during the first year, ${ }^{74}$ due to the nature of the remodeling cycle $^{41}$ this may be less relevant for changes in BMD.

2) Due to the rapid closing of training and assessment facilities combined with the partial lock-down in Bavaria, we were forced to conduct our retests within three days (ie, Friday to Sunday), moreover with only 1 day advance notice. Due to this narrow window, seven participants were unable to join the tests. Although we applied ITT with the imputation of missing values and thus reached the calculated sample size of 25 participants per group, there is some evidence that we lost power compared with the calculation of a full dataset. ${ }^{75} 3$ ) Unlike pharmaceutic agents, exercise is a comprehensive tool which, if properly designed and applied, might positively address a large variety of biomedical variables. Thus, it is challenging to design and evaluate exercise protocols that aimed to address several aims important for health and performance. Due to necessary compromises and the need ${ }^{76,77}$ for time-effective training protocols, some parameters might not be optimally addressed. As shown by corresponding systematic reviews/meta-analysis, this approach results in lower overall effects; furthermore, the statistical procedures for handling multiplicity are difficult and debatable. 4) We introduced an active (attention) control group to give women in the $\mathrm{CG}$ an opportunity to exercise and so, without being too apparent, prevent those women from doing uncontrolled exercise with potentially more bone-specific effects. Considering that a waiting control group is not an option for an 18-month study, we opted for full communication of pros and cons of both groups, which can be considered as the reason why 21 women refused to be randomly assigned to the groups. Despite the low-intensity and low volume exercise protocol of the $\mathrm{CG}$, considering the widely untrained status of our women, the exercise protocol may have affected some of the study endpoints addressed. Thus, we speculate that some changes between groups (eg, performance parameters, menopausal complaint) might be less pronounced compared to an approach with an "inactive" CG. 5) Baseline Vitamin $\mathrm{D}$ levels of both groups were below the recommended concentration but significantly improved after 13 months of supplementation. As studies have shown a positive effect of vitamin D supplementation on bone density, ${ }^{78}$ there is some evidence that Vit-D supplementation might have contributed to our result.

Finally, it is difficult to endorse an exercise protocol that had to be terminated after 13 of the planned 18 months and might therefore not have fully generated its potential effect - particularly on BMD. The advantages of the exercise protocol are its attractiveness, feasibility and safety. In summary, however, we have added further evidence that a multipurpose exercise program positively affects a multitude of risk factors and complaints related to the menopausal transition or increasing age.

\section{Data Sharing Statement}

The data that support the findings of this study are available from the corresponding author $(\mathrm{MH})$, upon reasonable request.

\section{Acknowledgment}

The present work was performed in (partial) fulfillment of the requirements for obtaining the degree "Dr. rer. biol. hum" for the first author.

This study is one of the intellectual outputs of the project "ACTLIFE-Physical activity the tool to improve the quality of life in osteoporosis people" and had grant support from the European Union's Erasmus Plus Sport program under grant agreement No. 2017-2128/001-001. We further acknowledge support by Deutsche Forschungsgemeinschaft and Friedrich-Alexander-University Erlangen-Nürnberg within the funding program "Open Access Publishing". However, the funding sources had no role in the design of this study nor its execution, analyses, interpretation of the data, or decision to submit results.

\section{Author Contributions}

All authors contributed to data analysis, drafting or revising the article, have agreed on the journal to which the article will be submitted, gave final approval of the version 
to be published, and agree to be accountable for all aspects of the work.

Conceptualization: all authors significantly contribute to the conceptualization of the study.

Data curation: $\mathrm{MH}, \mathrm{MS}, \mathrm{MG}, \mathrm{WK}$

Formal analysis: $\mathrm{MH}, \mathrm{MK}, \mathrm{WK}$

Investigation: $\mathrm{MH}, \mathrm{SvS}, \mathrm{MS}, \mathrm{MG}, \mathrm{WK}$

Methodology: MH, MK, WK

Project administration: $\mathrm{MH}, \mathrm{MS}$

Resources: all authors significantly contributed to gain resources for the project.

Supervision: $\mathrm{MH}, \mathrm{SvS}, \mathrm{MS}, \mathrm{MG}, \mathrm{WK}$

Validation: $\mathrm{MH}, \mathrm{MK}, \mathrm{WK}^{79}$

Visualization: not applicable

Writing - original draft: $\mathrm{MH}, \mathrm{WK}$

Writing - review and editing: all authors significantly contribute to writing, review and editing.

\section{Disclosure}

The authors report no conflicts of interest in this work.

\section{References}

1. Hoga L, Rodolpho J, Goncalves B, Quirino B. Women's experience of menopause: a systematic review of qualitative evidence. JBI Database System Rev Implement Rep. 2015;13(8):250-337. doi:10. 11124/01938924-201513080-00018

2. Overlie I, Moen MD, Morkrid L. The endocrine transition around menopause - a five year prospective study with profiles of gonadotropins, estrogens, androgens and SHBG among healthy women. Acta Obstet Gynecol Scand. 1999;78:642-647.

3. McKinlay SM. The normal menopause transition: an overview. Maturitas. 1996;23(2):137-145. doi:10.1016/0378-5122(95)009 85-X

4. Finkelstein JS, Brockwell SE, Mehta V, et al. Bone mineral density changes during the menopause transition in a multiethnic cohort of women. J Clin Endocrinol Metab. 2008;93(3):861-868. doi:10.1210/ jc. 2007-1876

5. Aloia JF, McGowan DM, Vaswani AN, Ross PL, Cohn SH. Relationship of menopause to skeletal and muscle mass. Am J Clin Nutr. 1991;53:1378-1383.

6. Sowers M, Zheng H, Tomey K, et al. Changes in body composition in women over six years at midlife: ovarian and chronological aging. J Clin Endocrinol Metab. 2007;92(3):895-901. doi:10.1210/jc.20061393

7. Maltais ML, Desroches J, Dionne IJ. Changes in muscle mass and strength after menopause. J Musculoskelet Neuronal Interact. 2009;9 (4):186-197.

8. Sirola J, Rikkonen T. Muscle performance after the menopause. $\mathrm{J} \mathrm{Br}$ Menopause Soc. 2005;11(2):45-50. doi:10.1258/136218005775544 561

9. Nelson HD. Menopause. Lancet. 2008;371(9614):760-770. doi:10.1016/S0140-6736(08)60346-3

10. Gao H-L, Gao H-X, Sun FM, Zhang L. Effects of walking on body composition in perimenopausal and postmenopausal women: a systematic review and meta-analysis. Menopause. 2016;23(8): 928-934. doi:10.1097/GME.0000000000000627
11. Howe TE, Shea B, Dawson LJ, et al. Exercise for preventing and treating osteoporosis in postmenopausal women. Cochrane Database Syst Rev. 2011;(7):CD000333.

12. Lobo RA, Davis SR, De Villiers TJ, et al. Prevention of diseases after menopause. Climacteric. 2014;17(5):540-556. doi:10.3109/136971 37.2014.933411

13. Slaven L, Lee C. Mood and symptom reporting among middle-aged women: the relationship between menopausal status, hormone replacement therapy, and exercise participation. Health Psychol. 1997;16 (3):203-208. doi:10.1037/0278-6133.16.3.203

14. Daley AJ, Stokes-Lampard HJ, Macarthur C. Exercise to reduce vasomotor and other menopausal symptoms: a review. Maturitas. 2009;63(3):176-180. doi:10.1016/j.maturitas.2009.02.004

15. Grindler NM, Santoro NF. Menopause and exercise. Menopause. 2015;22(12):1351-1358. doi:10.1097/GME.0000000000000536

16. Shojaa N, von Stengel S, Schoene D, et al. Effect of exercise training on bone mineral density in postmenopausal women: A systematic review and meta-analysis of intervention studies. Front Physiol. 2020;11:1427-1444. doi:10.3389/fphys.2020.00652

17. Kemmler W, Lauber D, Weineck J, Hensen J, Kalender W, Engelke K. Benefits of 2 years of intense exercise on bone density, physical fitness, and blood lipids in early postmenopausal osteopenic women: results of the Erlangen Fitness Osteoporosis Prevention Study (EFOPS). Arch Intern Med. 2004;164(10):1084-1091. doi:10.1001/archinte.164.10.1084

18. Kemmler W, Bebenek M, von Stengel S, Engelke K, Kalender WA. Effect of block-periodized exercise training on bone and coronary heart disease risk factors in early post-menopausal women: a randomized controlled study. Scand J Med Sci Sports. 2013;23 (1):121-129. doi:10.1111/j.1600-0838.2011.01335.x

19. World_Medical_Association. World Medical Association Declaration of Helsinki: ethical principles for medical research involving human subjects. JAMA. 2013;310(20):2191-2194. doi:10.1001/jama.2013.281053

20. DVO. Prophylaxe, Diagnostik und Therapie der OSTEOPOROSE bei postmenopausalen Frauen und bei Männern Stuttgart. Schattauer; 2017.

21. Gießing J. HIT-Hochintensitätstraining Arnsberg. Novagenics-Verlag; 2008.

22. Bebenek M, Kemmler W, von Stengel S, Engelke K, Kalender W. Effect of exercise and Cimicifuga racemosa (CR BNO 1055) on postmenopausal risk factors and complaints - the randomized controlled TRACE Study. Menopause. 2010;17(4):791-800.

23. Steele J, Fisher J, Giessing J, Gentil P. Clarity in reporting terminology and definitions of set end points in resistance training. Muscle Nerve. 2017;368-374(3):368-374. doi:10.1002/ mus. 25557

24. Zourdos MC, Klemp A, Dolan C, et al. Novel resistance training-specific rating of perceived exertion scale measuring repetitions in reserve. $J$ Strength Cond Res. 2016;30(1):267-275. doi:10.1519/JSC.0000000000001049

25. Kemmler WK, Lauber D, Wassermann A, Mayhew JL. Predicting maximal strength in trained postmenopausal woman. J Strength Cond Res. 2006;20(4):838-842.

26. Borg G, Borg E. The Borg CR Scales ${ }^{\circledR}$ Folder. Hasselby, Sweden; 2010.

27. Alberti KG, Zimmet P, Shaw J. Metabolic syndrome-a new worldwide definition. A consensus statement from the international diabetes federation. Diabet Med. 2006;23(5):469-480. doi:10.1111/ j.1464-5491.2006.01858.x

28. Johnson JL, Slentz CA, Houmard JA, et al. Exercise training amount and intensity effects on metabolic syndrome (from studies of a targeted risk reduction intervention through defined exercise). Am J Cardiol. 2007;100 (12):1759-1766. doi:10.1016/j.amjcard.2007.07.027

29. Hauser GA, Schneider HP, Rosemeier PJ, Potthoff P. Die Selbstbeurteilungs-Skala für klimakterischen Beschwerden (Menopause Rating Scale II). J Menopause. 1999;4:13-17. 
30. Kemmler W, Weineck J, Kalender WA, Engelke K. The effect of habitual physical activity, non-athletic exercise, muscle strength, and $\mathrm{VO} 2 \mathrm{max}$ on bone mineral density is rather low in early postmenopausal osteopenic women. J Musculoskelet Neuronal Interact. 2004;4 (3):325-334.

31. Schneider HP, Heinemann LA, Rosemeier HP, Potthoff P, Behre HM. The Menopause Rating Scale (MRS): reliability of scores of menopausal complaints. Climacteric. 2000;3(1):59-64. doi:10.3109/1369 7130009167600

32. Kemmler W, Engelke K, Lauber D, Weineck J, Hensen J, Kalender WA. Exercise effects on fitness and bone mineral density in early postmenopausal women: 1-year EFOPS results. Med Sci Sports Exerc. 2002;34(12):2115-2123. doi:10.1097/00005768-2002 12000-00038

33. R_Development_Core_Team R. A language and environment for statistical computing. Vienna, Austria: $R$ Foundation Statistical Computing. 2019.

34. Honaker J, King G, Blackwell M. Amelia II: A program for missing data. JSS. 2011;45(7):1-47.

35. Cohen J Statistical Power Analysis for the Behavioral Sciences. 2nd ed. Hillsdale NJ eds Lawrence Earlbaum Associate; 1988.

36. Karlamangla AS, Burnett-Bowie SM, Crandall CJ. Bone health during the menopause transition and beyond. Obstet Gynecol Clin North Am. 2018;45(4):695-708. doi:10.1016/j.ogc.2018.07.012

37. Beck BR, Daly RM, Singh MA, Taaffe DR. Exercise and Sports Science Australia (ESSA) position statement on exercise prescription for the prevention and management of osteoporosis. J Sci Med Sport. 2016;20(5):438-445. doi:10.1016/j.jsams.2016.10.001

38. Daly RM, Dalla Via J, Duckham RL, Fraser SF, Helge EW. Exercise for the prevention of osteoporosis in postmenopausal women: an evidence-based guide to the optimal prescription. Braz J Phys Ther. 2019;23(2):170-180. doi:10.1016/j.bjpt.2018.11.011

39. Kemmler W, von Stengel S. Exercise and osteoporosis-related fractures: perspectives and recommendations of the sports and exercise scientist. Physician Sportmed. 2011;39(1):142-157. doi:10.3810/ psm.2011.02.1872

40. Watson SL, Weeks BK, Weis LJ, Harding AT, Horan SA, Beck BR. High-intensity resistance and impact training improves bone mineral density and physical function in postmenopausal women with osteopenia and osteoporosis: the LIFTMOR randomized controlled trial. J Bone Miner Res. 2018;33(2):211-220. doi:10.1002/jbmr.3284

41. Eriksen EF. Cellular mechanisms of bone remodeling. Rev Endocr Metab Disord. 2010;11(4):219-227. doi:10.1007/s11154010-9153-1

42. Tong A, Flemming K, McInnes E, Oliver S, Craig J. Enhancing transparency in reporting the synthesis of qualitative research: ENTREQ. BMC Med Res Methodol. 2012;12:181. doi:10.1186/ 1471-2288-12-181

43. Yuan Y, Chen X, Zhang L, et al. The roles of exercise in bone remodeling and in prevention and treatment of osteoporosis. Prog Biophys Mol Biol. 2016;122(2):122-130. doi:10.1016/j.pbiomolbio. 2015.11.005

44. Kemmler W, von Stengel S. Exercise frequency, health risk factors, and diseases of the elderly. Arch Phys Med Rehabil. 2013;94 (11):2046-2053. doi:10.1016/j.apmr.2013.05.013

45. Kemmler W, von Stengel S, Kohl M. Exercise frequency and bone mineral density development in exercising postmenopausal osteopenic women. Is there a critical dose of exercise for affecting bone? Results of the erlangen fitness and osteoporosis prevention study. Bone. 2016;89:1-6. doi:10.1016/j.bone.2016.04.019

46. Ho SC, Wu S, Chan SG, Sham A. Menopausal transition and changes of body composition: a prospective study in Chinese perimenopausal women. Int $J$ Obes. 2010;34(8):1265-1274. doi:10.1038/ijo.2010.33
47. Poehlman ET, Toth MJ, Gardner AW. Changes in energy balance and body composition at menopause: a controlled longitudinal study. Ann Int Med. 1995;123(5):673-675. doi:10.7326/0003-4819-123-9-1995 11010-00005

48. Hagstrom AD, Marshall PW, Halaki M, Hackett DA. The effect of resistance training in women on dynamic strength and muscular hypertrophy: a systematic review with meta-analysis. Sports Med. 2020;50(6):1075-1093. doi:10.1007/s40279-019-01247-x

49. Schwingshackl L, Dias S, Strasser B, Hoffmann G. Impact of different training modalities on anthropometric and metabolic characteristics in overweight/obese subjects: a systematic review and network meta-analysis. PLoS One. 2013;8(12):e82853. doi:10.1371/journal. pone. 0082853

50. Stiegler P, Cunliffe A. The role of diet and exercise for the maintenance of fat-free mass and resting metabolic rate during weight loss. Sports Med. 2006;36(3):239-262. doi:10.2165/00007256-20063 6030-00005

51. Weck M, Bornstein SR, Barthel A, Bluher M. [Strategies for successful weight reduction - focus on energy balance]. Dtsch Med Wochenschr. 2012;137(43):2223-2228. German.

52. Lesser IA, Guenette JA, Hoogbruin A, et al. Association between exercise-induced change in body composition and change in cardiometabolic risk factors in postmenopausal South Asian women. Appl Physiol Nutr Metab. 2016;41(9):931-937. doi:10.1139/apnm-2016-0082

53. Nicklas BJ, Wang X, You T, et al. Effect of exercise intensity on abdominal fat loss during calorie restriction in overweight and obese postmenopausal women: a randomized, controlled trial. Am J Clin Nutr. 2009;89(4):1043-1052. doi:10.3945/ajen.2008.26938

54. Mandrup CM, Egelund J, Nyberg M, et al. Effects of high-intensity training on cardiovascular risk factors in premenopausal and postmenopausal women. Am J Obstet Gynecol. 2017;216(4):384e381384 e311. doi:10.1016/j.ajog.2016.12.017

55. Bateman LA, Slentz CA, Willis LH, et al. Comparison of aerobic versus resistance exercise training effects on metabolic syndrome (from the studies of a targeted risk reduction intervention through defined exercise - STRRIDE-AT/RT). Am J Cardiol. 2011;108 (6):838-844. doi:10.1016/j.amjcard.2011.04.037

56. Conceicao MS, Bonganha B, Vechin V, et al. Sixteen weeks of resistance training can decrease the risk of metabolic syndrome in healthy postmenopausal women. Clin Interv Aging. 2013;8:12 21-1228. doi:10.2147/CIA.S44245

57. Dieli-Conwright CM, Courneya KS, Demark-Wahnefried W, et al. Effects of aerobic and resistance exercise on metabolic syndrome, sarcopenic obesity, and circulating biomarkers in overweight or obese survivors of breast cancer: a randomized controlled trial. $J$ Clin Oncol. 2018;36(9):875-883. doi:10.1200/JCO.2017.75.7526

58. Earnest CP, Johannsen NM, Swift DL, et al. Aerobic and strength training in concomitant metabolic syndrome and type 2 diabetes. Med Sci Sports Exerc. 2014;46(7):1293-1301. doi:10.1249/MSS.000000 0000000242

59. Kemmler W, von Stengel S, Bebenek M, Kalender WA. Long-term exercise and risk of metabolic and cardiac diseases: the erlangen fitness and prevention study. Evidence-Based Complementary Alternative Med. 2013;2013:768431. doi:10.1155/2013/768431

60. Kemmler W, Kohl M. Effects of high intensity resistance training versus whole-body electromyostimulation on cardiometabolic risk factors in untrained middle aged males. A randomized controlled trial. J Sports Res. 2016;3(2):44-55. doi:10.18488/journal.90/2016. 3.2/90.2.44.55

61. Kemmler W, Wittke A, Bebenek M, Fröhlich M, von Stengel S. High intensity resistance training methods with and without protein supplementation to fight cardiometabolic risk in middle-aged males A randomized controlled trial. Biomed Res Int. 2016;2016:1-9. doi:10.1155/2016/9705287 
62. Tomeleri CM, Souza MF, Burini RC, et al. Resistance training reduces metabolic syndrome and inflammatory markers in older women: A randomized controlled trial. J Diabetes. 2016;3(2):3 28-337. doi:10.1111/1753-0407.12614

63. Wittmann K, Sieber C, von Stengel S, et al. Impact of whole body electromyostimulation on cardiometabolic risk factors in older women with sarcopenic obesity: the randomized controlled FORMOsA-sarcopenic obesity study. Clin Interv Aging. 2016;11: 1697-1706. doi:10.2147/CIA.S116430

64. Mirzaiinjmabadi K, Anderson D, Barnes M. The relationship between exercise, Body Mass Index and menopausal symptoms in midlife Australian women. International J Nursing Practice. 2006;12 (1):28-34. doi:10.1111/j.1440-172X.2006.00547.x

65. Perez-Lopez FR, Martinez-Dominguez SJ, Lajusticia H, Chedraui P. Health Outcomes Systematic Analyses P. Effects of programmed exercise on depressive symptoms in midlife and older women: A meta-analysis of randomized controlled trials. Maturitas. 2017;106:38-47. doi:10.1016/j.maturitas.2017.09.001

66. Daley A, Stokes-Lampard H, Thomas A, MacArthur C. Exercise for vasomotor menopausal symptoms. Cochrane Database Syst Rev. 2014;(11):CD006108.

67. Rubio-Arias JA, Marin-Cascales E, Ramos-Campo DJ, Hernandez AV, Perez-Lopez FR. Effect of exercise on sleep quality and insomnia in middle-aged women: A systematic review and meta-analysis of randomized controlled trials. Maturitas. 2017;100:49-56. doi:10.1016/j.maturitas.2017.04.003

68. Bassey EJ, Ramsdale SJ. Weight-bearing exercise and ground reaction forces: a 12-month randomized controlled trial of effects on bone mineral density in healthy postmenopausal women. Bone. 1995;16(4):469-476.

69. Bemben DA, Fetters NL, Bemben MG, Nabavi N, Koh ET. Musculoskeletal responses to high- and low-intensity resistance training in early postmenopausal women. Med Sci Sports Exerc. 2000;32(11):1949-1957. doi:10.1097/00005768200011000-00020

70. Chilibeck PD, Davison KS, Whiting SJ, Suzuki Y, Janzen CL, Peloso P. The effect of strength training combined with bisphosphonate (etidronate) therapy on bone mineral, lean tissue, and fat mass in postmenopausal women. Can J Physiol Pharmacol. 2002;80 (10):941-950. doi:10.1139/y02-126
71. Figueroa A, Going SB, Milliken LA, et al. Effects of exercise training and hormone replacement therapy on lean and fat mass in postmenopausal women. J Gerontol Biol Sci Med Sci. 2003;58(3):266-270. doi:10.1093/gerona/58.3.M266

72. Humphries B, Newton RU, Bronks R, et al. Effect of exercise intensity on bone density, strength, and calcium turnover in older women. Med Sci Sports Exerc. 2000;32(6):1043-1050. doi:10.1097/ 00005768-200006000-00002

73. Pruitt LA, Jackson RD, Bartels RL, Lehnhard HJ. Weight-training effects on bone mineral density in early postmenopausal women. J Bone Miner Res. 2009;7(2):179-185. doi:10.1002/jbmr.5650070209

74. Kemmler W, Kohl M, Froehlich M, Engelke K, von Stengel S, Schoene D. Effects of high intensity resistance training on fitness and fatness in older men with osteosarcopenia. final results of the randomized controlled franconian osteopenia and sarcopenia trial (FrOST). Front Physiol. 2020;11. doi:10.3389/fphys.2020.01014

75. Zha R, Harel O. Power calculation in multiply imputed data. J Statistical Papers. 2019;1-27.

76. Rütten A, Abu-Omar K, Lampert T, Ziese T Körperliche Aktivität [Physical Activity]. Report. Berlin: Statistisches Bundesamt;2005.

77. Carlson SA, Fulton JE, Schoenborn CA, Loustalot F. Trend and prevalence estimates based on the 2008 physical activity guidelines for Americans. Am J Prev Med. 2010;39(4):305-313. doi:10.1016/j. amepre.2010.06.006

78. Reid IRB, Grey MJ. A. Effects of vitamin D supplements on bone mineral density: a systematic review and meta-analysis. Lancet. 2014;383.

79. Abadie BR, Altorfer GL, Schuler PB. Does a regression equation to predict maximal strength in untrained lifters remain valid when the subjects are technique trained? J Strength Cond Res. 1999;13(3):259-263.

\section{Publish your work in this journal}

Clinical Interventions in Aging is an international, peer-reviewed journal focusing on evidence-based reports on the value or lack thereof of treatments intended to prevent or delay the onset of maladaptive correlates of aging in human beings. This journal is indexed on PubMed Central, MedLine, CAS, Scopus and the Elsevier
Bibliographic databases. The manuscript management system is completely online and includes a very quick and fair peer-review system, which is all easy to use. Visit http://www.dovepress.com/ testimonials.php to read real quotes from published authors. 